\title{
INFLUENCE OF THE SPATIAL VARIABILITY OF JOINTS CHARACTERISTICS ON THE ELASTIC PROPERTIES OF MASONRY
}

\author{
MARIA LAURA DE BELLIS ${ }^{1 *}$, VINCENZO SEPE ${ }^{1}$ AND MARCELLO VASTA ${ }^{1}$ \\ ${ }^{1}$ Department of Engineering and Geology (INGEO) \\ Università "G. D'Annunzio" di Chieti - Pescara \\ Viale Pindaro 42, 65127 Pescara, Italy \\ e-mail: \{marialaura.debellis, vincenzo.sepe, marcello.vasta\}@unich.it, (*corresponding author)
}

Keywords: Masonry Structures, Homogenization, Random Uncertainties

\begin{abstract}
Brick masonry is a multicomponent composite material, characterized by a marked inhomogeneity, a highly anisotropic behavior arising from the complex interactions between joints and bricks, and a considerable spatial variability of material properties, particularly relevant in the case of historical masonries. The focus of this paper is on the interdependence between the spatial variability of mechanical properties of joints and the overall homogenized response of a given volume element of masonry. It is assumed that mechanical properties of bed joints may vary according to a fixed probability distribution and correlation function and the mechanical response of the volume element is investigated in terms of the overall elastic stiffness. As confirmed by preliminary results, the spatial variability of the mechanical properties may affect the overall response of a masonry wall.
\end{abstract}

\section{INTRODUCTION}

Experimental and numerical results [1-3], referred either to single constituents or to fullsized masonry walls, have shown remarkable spatial variations of constitutive variables, i.e. Young modulus, tensile and compressive strength among others, also in case of new structures, as well as geometrical variations of the masonry arrangement [4]. In the case of historical masonries, such variability can cause even more relevant effects, especially due to the role of ageing processes, that can locally affect the mechanical characteristics of bed and head mortar joints.

Based on the aforementioned considerations, the focus of this paper is on the interdependence between the spatial variability of mechanical properties of joints and the overall response of a given volume element of the masonry. To this aim, a well established computational homogenization technique is combined with a properly conceived stochastic procedure. It is assumed that elastic properties of bed joints may vary according to a fixed probability distribution and correlation function. The mechanical response, in terms of overall elastic stiffness of the selected volume element, is investigated as a function of mean, variance and correlation length of the elastic properties of the joints, in a realistic range according to values experimentally observed. 


\section{ELASTIC PROPERTIES OF A VOLUME ELEMENT}

Although geometrical and mechanical characteristics of both components of a given volume element and their related uncertainties may play a relevant role on the overall behavior, the most pronounced uncertainties on the global elastic behavior of masonry are likely due to available information about deformability of joints and its spatial variability.

To this aim, for the volume element (Fig. $1, \mathrm{~L}_{0}=26 \mathrm{~cm}$ ) of a regular masonry made of bricks and mortar joints with running bond arrangement, the sensitivity of homogenized elastic properties is investigated as a function of mechanical characteristics of bed mortar joints, varying with a given standard deviation $\sigma_{\mathrm{E}}$ around a mean value $\mu_{\mathrm{E}}$. A constant value $\nu=0.2$ has instead been assigned to the Poisson coefficient of joints and elastic parameters of bricks have also been considered as constant, $\mathrm{E}_{\mathrm{b}}=6000 \mathrm{MPa}$ and $v_{\mathrm{b}}=0.2$.

Each bed joint (with a total length $L_{0}$, see Fig. 1) is discretized in 10 parts $(\Delta x=2.6 \mathrm{~cm})$, assigning to each of them the elastic modulus $\mathrm{E}$ in the mean point, as obtained by a random generation of $\mathrm{E}(\mathrm{x})$ (see Sect. 3.2) in the interval $0 \leq \mathrm{x} \leq \mathrm{L}$, with $\mathrm{L} \gg \mathrm{L}_{0}$ and a sampling step $\delta \mathrm{x}=\Delta \mathrm{x} / 100$.

In the numerical investigation described below a mean value $\mu_{\mathrm{E}}=500 \mathrm{MPa}$ was assumed, with standard deviation varying between $\sigma_{\mathrm{E}}=0.07 \mu_{\mathrm{E}}$ and $\sigma_{\mathrm{E}}=0.35 \mu_{\mathrm{E}}$ and six different correlation lengths $\left(\mathrm{L}_{\mathrm{c} 1}=0.26 \mathrm{~cm}, \mathrm{~L}_{\mathrm{c} 2}=0.65 \mathrm{~cm}, \mathrm{~L}_{\mathrm{c} 3}=1.3 \mathrm{~cm}, \mathrm{~L}_{\mathrm{c} 4}=2.6 \mathrm{~cm}, \mathrm{~L}_{\mathrm{c} 5}=5.2 \mathrm{~cm}, \mathrm{~L}_{\mathrm{c} 6}=13\right.$ $\mathrm{cm})$.

Namely, for a given $\sigma_{E}$ and correlation length $\mathrm{L}_{\mathrm{c}}$, a random realization of $\mathrm{E}(\mathrm{x})$ was obtained

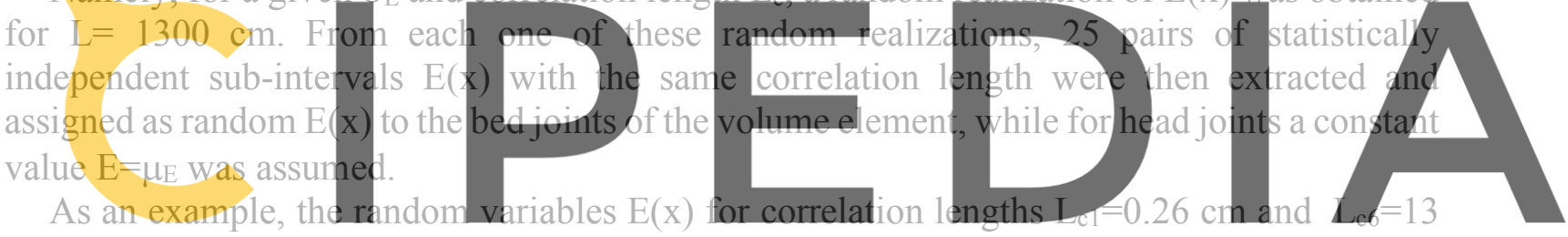
$\mathrm{cm}$ are reported in Fig. 2.

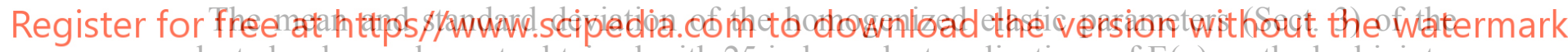
selected volume element, obtained with 25 independent realizations of $\mathrm{E}(\mathrm{x})$ on the bed joints, are reported in Sect. 3.2 as a function of standard deviation $\sigma_{\mathrm{E}}$ and correlation length $\mathrm{L}_{\mathrm{c}}$.
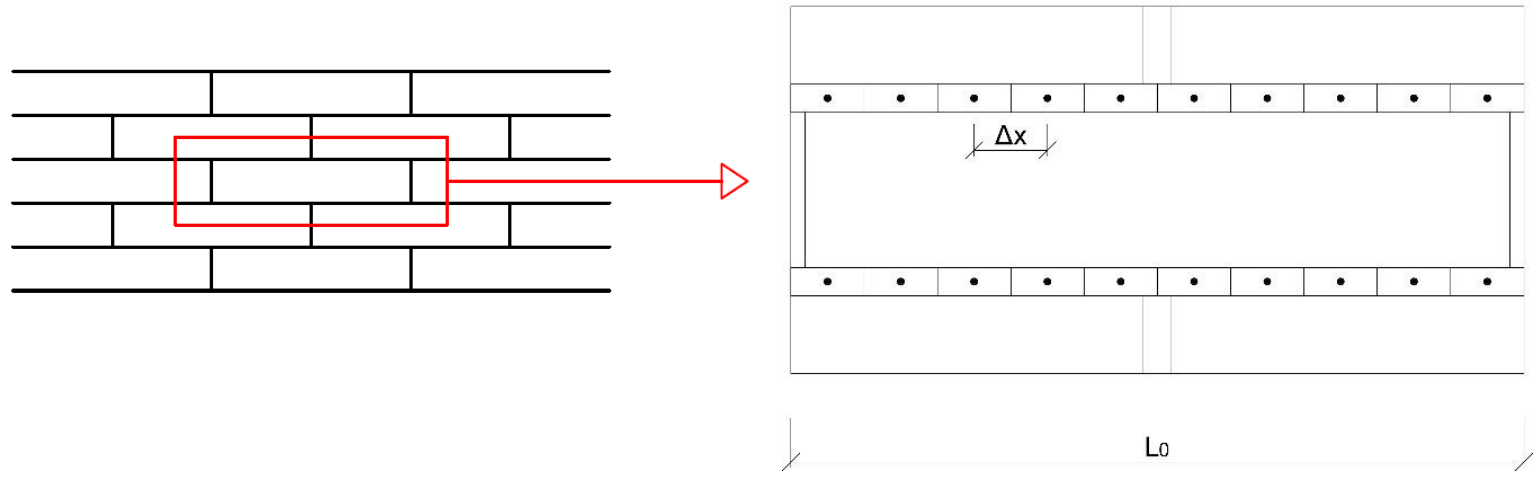

Figure 1: selected volume element: $\mathrm{L}_{0}=26 \mathrm{~cm}$ 

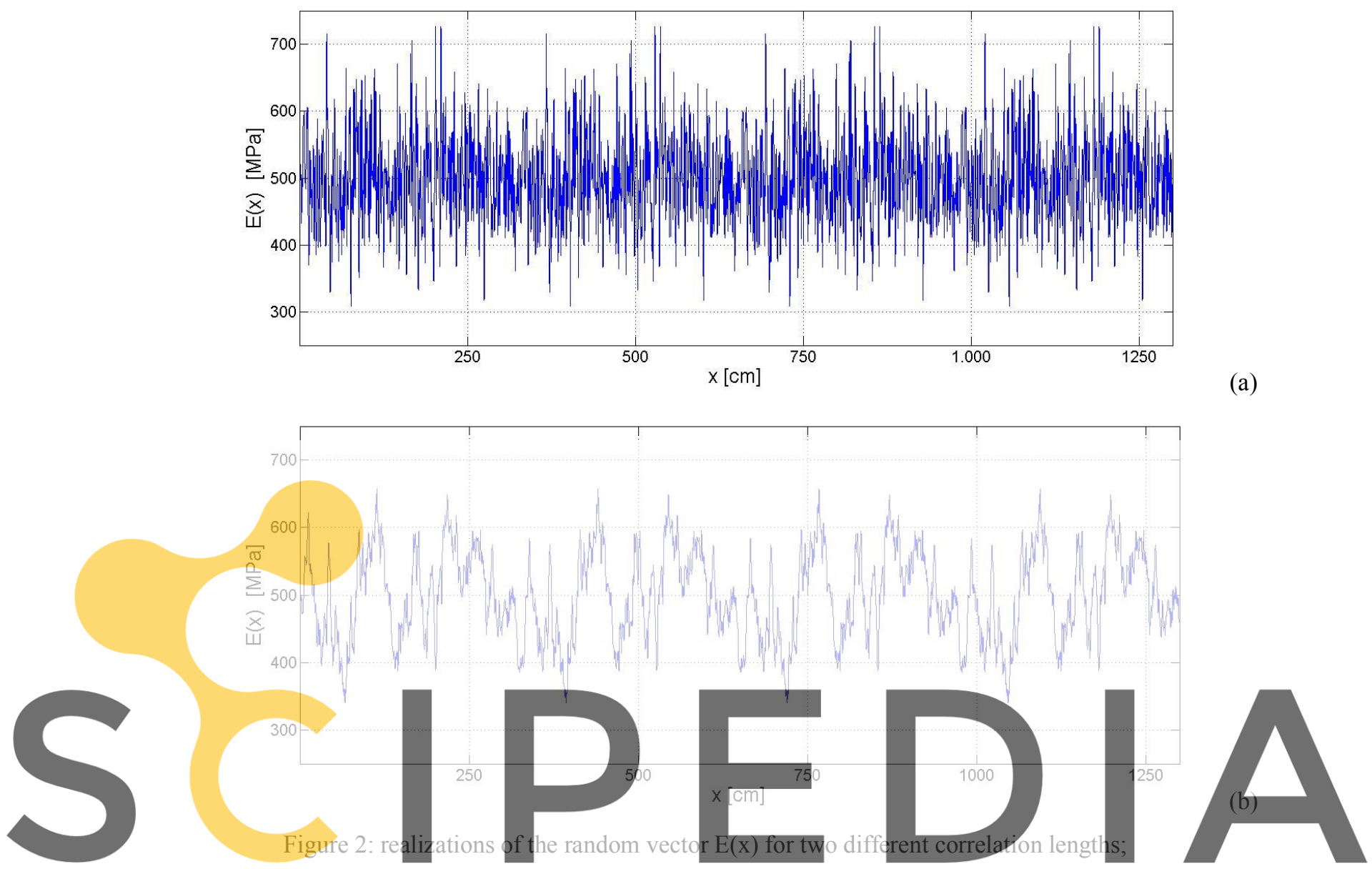

a) $\mathrm{L}_{\mathrm{cl}}=0.26 \mathrm{~cm}$; b) $\mathrm{L}_{\mathrm{c} 6}=13 \mathrm{~cm}$

Register for free at https//www scipedia.com to download the version without the watermark

The homogenization of the masonry material is performed resorting to a first order computational homogenization technique. Consistently with the classical approach [5-6], the composite material is described at two scales of interest, the microscopic and the macroscopic scale, as a Cauchy continuum. At the microscopic scale, the actual material is thoroughly described, in terms of geometric and constitutive parameters characterizing both blocks and mortar joints, while at the macroscopic one the heterogeneous material is ideally replaced by an equivalent homogeneous material, able to reproduce the overall elastic response of the composite material.

The classical first order multiscale scheme is briefly recalled in the framework of a strain driven approach. FE elastic analyses are exploited to evaluate the effective elastic properties of the masonry characterized by spatial variability of the elastic constants in the mortar joints.

A one to one correspondence is established between a macroscopic point of the first order homogenized continuum and the corresponding material portion at the microscopic scale.

At the typical material point $\mathbf{X}=\{X, Y\}^{T}$ at the macroscopic level, the displacement vector $\mathbf{U}=$ $\{U, V\}^{T}$ is defined and the strain vector results as 


$$
\boldsymbol{E}=\left\{\begin{array}{l}
E_{X X} \\
E_{Y Y} \\
\Gamma_{X Y}
\end{array}\right\}=\mathbf{D}\left\{\begin{array}{l}
U \\
V
\end{array}\right\}, \quad \text { where } \quad \mathbf{D}=\left[\begin{array}{cc}
r_{, X} & 0 \\
0 & { }^{\prime}, \\
{ }^{\prime}, Y & { }^{,} X
\end{array}\right],
$$

with $\mathbf{D}$ being the kinematic operator.

The overall linear elastic constitutive equations in the general anisotropic case depends on the overall elasticity tensor $\mathbf{C}$ as

$$
\boldsymbol{\Sigma}=\left\{\begin{array}{l}
\Sigma_{X X} \\
\Sigma_{Y Y} \\
T_{X Y}
\end{array}\right\}=\mathbf{C}\left\{\begin{array}{l}
E_{X X} \\
E_{Y Y} \\
\Gamma_{X Y}
\end{array}\right\}, \quad \text { where } \quad \mathbf{C}=\left[\begin{array}{lll}
C_{X X X X} & C_{X X Y Y} & C_{X X X Y} \\
C_{X X Y Y} & C_{Y Y Y Y} & C_{Y Y X Y} \\
C_{X X X Y} & C_{Y Y X Y} & C_{X Y X Y}
\end{array}\right]
$$

On the other hand, at the microscopic scale the corresponding strain $\varepsilon$ and stress $\sigma$ measures are similarly defined together with the local elasticity matrix $\mathbf{c}$.

In the case of a periodic continuum the vector $E$ is used as input quantity for the periodic cell and a properly defined the microscopic level. possible to detect the as the components of $\mathrm{C}$ by Nevertheless, in the case at hand, owing to
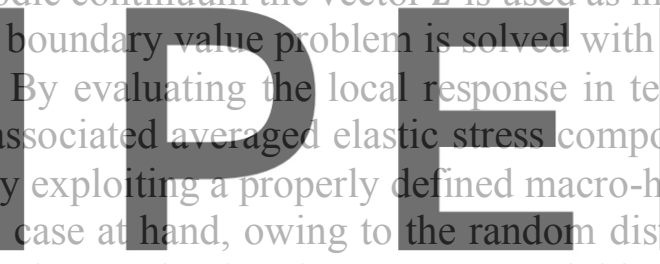

along the mortar joints, the running bond masonry material is only characterized by geometric

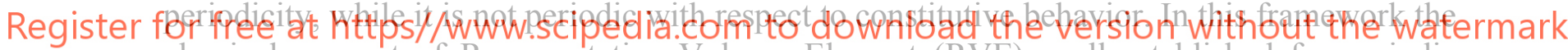
classical concept of Representative Volume Element (RVE), well established for periodic heterogeneous media, loses its validity. The RVE cannot be a-priori recognized and becomes itself an unknown of the problem, together with the estimation of the homogenized elastic moduli, i.e. the components of $\mathbf{C}$.

The RVE can be approached by using a finite size scaling of the so-called statistical volume element (SVE) [7-8] through two hierarchies of constitutive bounds, stemming from the solution of Dirichlet and Neuman boundary value problems at the microscopic level, respectively. The key idea is considering a trial portion of the heterogeneous material of a given characteristic size and keep enlarging such portion until the solution of both of Dirichlet and Neuman boundary value problems have fulfilled a properly defined convergence criterion.

The Dirichlet boundary conditions imposed along the boundary of the SVE can be written as

$$
\begin{aligned}
& u=E_{X X} x+\Gamma_{X Y} y \\
& v=\Gamma_{X Y} x+E_{Y Y} y
\end{aligned},
$$

while the Neumann boundary conditions result as 


$$
\begin{aligned}
& t_{x}=\Sigma_{X X} n_{x}+T_{X Y} n_{y} \\
& t_{y}=T_{X Y} n_{x}+\Sigma_{Y Y} n_{y}
\end{aligned}
$$

with $\left(t_{x}, t_{y}\right)$ the components of tractions and $\left(n_{x}, n_{y}\right)$ the components of the outward normal.

The macroscopic stress $\boldsymbol{\Sigma}$ and strain $\boldsymbol{E}$ measures are linked to the respective microscopic ones $\boldsymbol{\sigma}$ and $\boldsymbol{\varepsilon}$ through the so-called spatial averages, as follows

$$
\boldsymbol{\Sigma}=\frac{1}{\omega} \int_{\omega} \boldsymbol{\sigma} d \omega, \quad \boldsymbol{E}=\frac{1}{\omega} \int_{\omega} \boldsymbol{\varepsilon} d \omega
$$

Finally, the homogenized moduli can be obtained via the macro-homogeneity condition, accounting for infinitesimal deformation gradients. Such well-known condition establishes a correspondence between the average internal work over a portion of material at the microscopic level and the mechanical internal work density at the macroscopic point, as

$$
\boldsymbol{E}^{T} \Sigma=\frac{1}{\omega} \int_{\omega} \varepsilon^{T} \sigma d \omega
$$
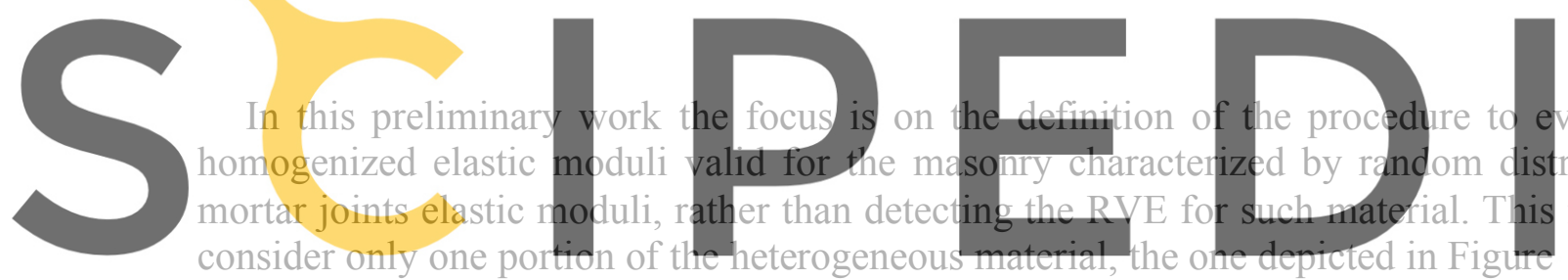
consider only one portion of the heterogeneous m

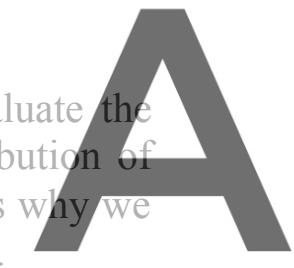

Furthermore, in order to obtain a synthetic description of the degree and direction of

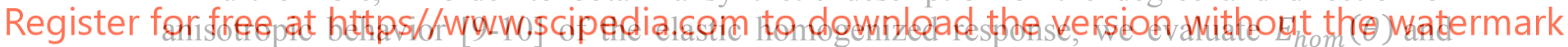

$v_{\text {hom }}(\theta)$ as a function of the counterclockwise angle $\theta$ between the standard basis $\left(\boldsymbol{e}_{x}, \boldsymbol{e}_{y}\right)$ and the basis $\left(\boldsymbol{a}_{x}, \boldsymbol{a}_{y}\right)$ rotated of $\theta$ about an axis through the origin. More specifically, $E_{\text {hom }}(\theta)$ and $v_{\text {hom }}(\theta)$ are obtained for tension applied along the direction identified by the unit vector $\boldsymbol{a}_{x}$ inclined at an angle $\theta$ with respect to $\boldsymbol{e}_{x}$.

The homogenized elastic compliance tensor $\boldsymbol{D}=\boldsymbol{C}^{-1}$ in the rotated reference system becomes $\boldsymbol{D}^{\theta}$ and the arising angular variation of the Young modulus and of the Poisson's ratio are consistently evaluated as

$$
E_{\text {hom }}(\theta)=\frac{1}{D_{X X X X}^{\theta}}, \quad v_{\text {hom }}(\theta)=-\frac{D_{X X Y Y}^{\theta}}{D_{X X X X}^{\theta}}
$$

where $D_{X X X X}^{\theta}$ and $D_{X X Y Y}^{\theta}$ are the components of $\boldsymbol{D}^{\theta}$.

The graphical representation of $E_{\text {hom }}(\theta)$ and $v_{\text {hom }}(\theta)$ versus $\theta$ is very suitable for an interesting physical interpretation. If the material is isotropic, indeed, the polar plot has the form 
of a unitary circle, while deviations from a perfect circle evince the degree and direction of anisotropic behavior.

\subsection{Reference case: homogeneous elastic properties of bed and head joints}

As a reference case, a constant value $\mathrm{E}(\mathrm{x})=\mu_{\mathrm{E}}$ has been assigned to both head and bed joints. The variation of Young modulus $\mathrm{E}_{0}(\theta)$ and Poisson coefficient $v(\theta)$ as a function of the angle $\theta$ are reported in Figure 3(a) and 3(b), respectively.

As shown by such figures, the equivalent stiffness of the selected volume element attains maximum and minimum values for $\theta=0$ (stress-strain parallel to the bed joints) and $\theta=\pi / 2$ (stress-strain orthogonal to the bed joints), respectively, while maximum values of Poisson coefficient are attained in pseudo-diagonal directions.
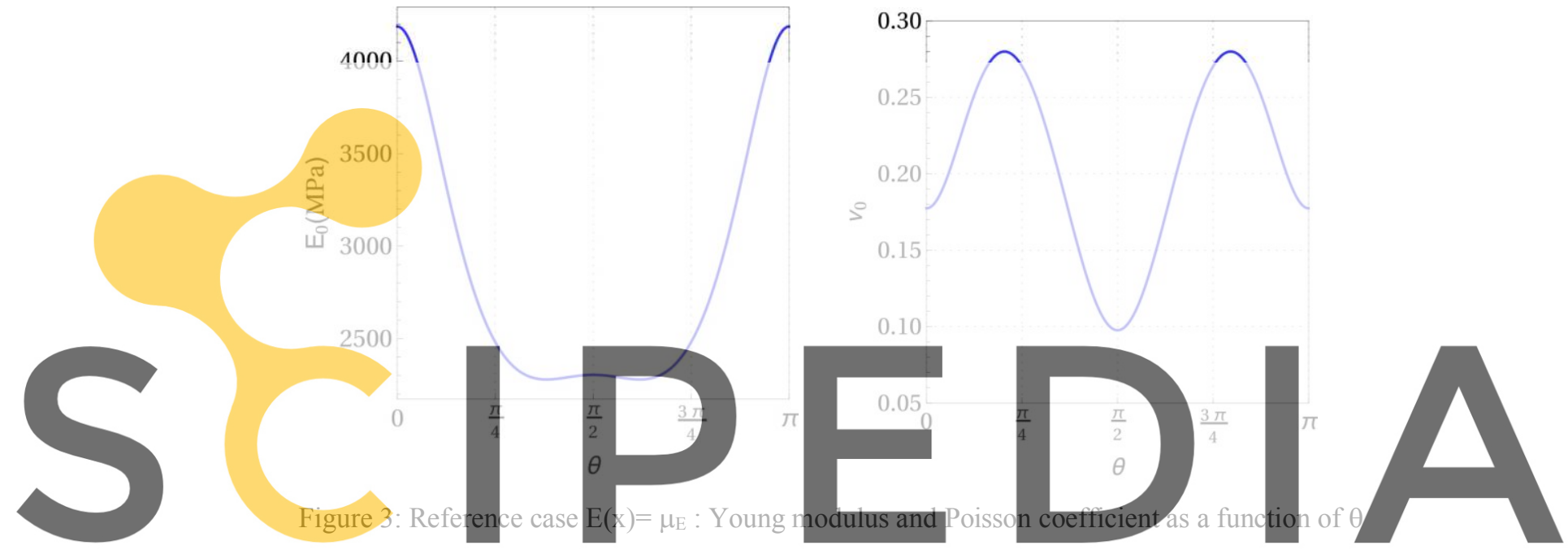

\subsection{Random elastic properties of bed joints} Register for free at https//Www, scipedia.com to download the version without the watermark Stationary Gaussian realizations of the Young modulus

$$
E(x)=\bar{E}+e(x)
$$

have been generated with $\bar{E}$ the mean value and $e(x)$ a stationary zero mean process obtained as the output of a first order linear scalar stochastic differential equation (Ornstein - Uhlenbeck process) [11]

$$
\frac{d e(x)}{d x}=-\lambda e(x)+W(x)
$$

where $\lambda>0$ and $W(x)$ a zero mean Gaussian stationary white noise input process with constant spectral density $S_{\mathrm{w}}(\omega)=S_{0}$ and correlation functions $R_{\mathrm{w}}(l)=\langle W(x) W(x+l)\rangle=2 \pi S_{0} \delta(l)$, where $\delta(\cdot)$ stands for the Dirac's delta distribution. 
The spectral density $S_{e}(\omega)$ and the covariance function $C_{e}(l)$ of the stationary process $e(x)$ are as follows

$$
\begin{gathered}
S_{e}(\omega)=\frac{2 \pi S_{0}}{\omega^{2}+\lambda^{2}} \\
C_{e}(l)=\langle e(x) e(x+l)\rangle=\frac{\pi S_{0}}{\lambda} \exp (-\lambda|l|)
\end{gathered}
$$

For the same input white noise $W(x)$ different correlated random realizations of $E(x)$ have been obtained for different values of the parameter $\lambda$ and intensity $S_{0}$.

The correlation length $L_{\mathrm{c}}$ is the inverse of $\lambda\left(C_{E}(x)=C_{e}(x)\right)$

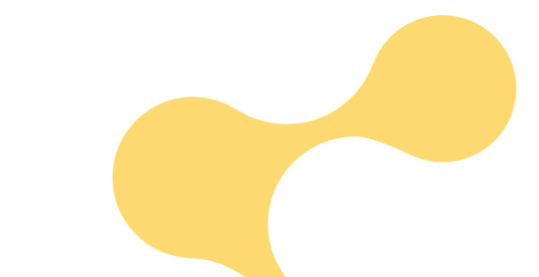

$$
L_{C}=\frac{1}{C_{E}(0)} \int_{0}^{+\infty} C_{E}(l) d l=\frac{1}{\lambda}
$$
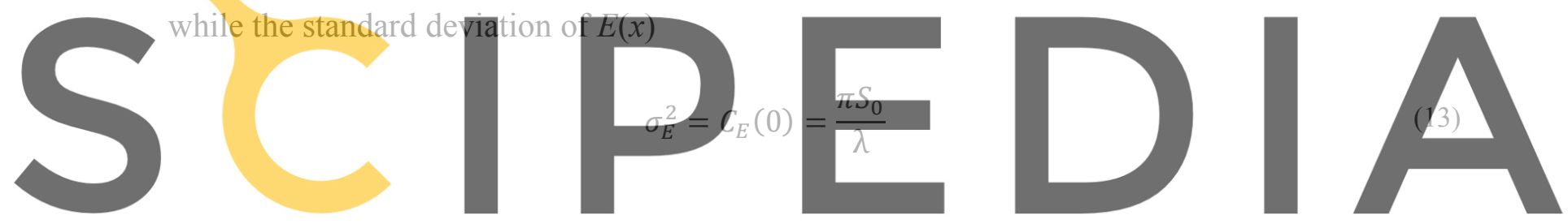

\section{Register for free at https//www.scipedia.com to download the version without the watermark}

As described in Sect.2, for each standard deviation $\sigma_{\mathrm{E}}$ and correlation length $\mathrm{L}_{\mathrm{c}} 25$ independent realizations of the elastic modulus $\mathrm{E}(\mathrm{x})$ of the bed joints have been considered for the volume element in Fig.1.

For each case the elastic tensor of the volume element has been obtained with the homogenization procedure described in Sect. 3 and boundary conditions of the Dirichlet type.

The mean values of the homogenized elastic modulus $E_{\text {hom }}(\theta)$ and Poisson coefficient $v_{\text {hom }}(\theta)$ in the considered set of 25 independent realizations almost coincide, as expected, with the values $\mathrm{E}_{0}(\theta)$ and $v_{0}(\theta)$ of the reference case (Sect. 3.1), independently from the correlation length $\mathrm{L}_{\mathrm{c}}$ or the variation coefficient $\mathrm{C}_{\mathrm{V}}=\sigma_{\mathrm{E}} / \mu_{\mathrm{E}}$. Therefore they are not reported here.

A significant dependence has instead been found for the standard deviation $\sigma_{\text {Ehom }}(\theta)$ and $\sigma_{\text {vhom }}(\theta)$ of the homogenized elastic modulus $\mathrm{E}_{\text {hom }}(\theta)$ and Poisson coefficient $v_{\text {hom }}(\theta)$.

Standard deviations of $\mathrm{E}_{\mathrm{hom}}(\theta)$ and $v_{\text {hom }}(\theta)$, normalized with respect to the values $\mathrm{E}_{0}(\theta)$ and $v_{0}(\theta)$ of the reference case (constant $E(x)=\mu_{E}$, Sect. 3.1) are described below for each angle $\theta$ as a function of the correlation length $\mathrm{L}_{\mathrm{c}}$ (Fig. 4a, 5a) and of the variation coefficient $\mathrm{C}_{\mathrm{V}}=\sigma_{\mathrm{E}} / \mu_{\mathrm{E}}$ of the random variable E(x) (Fig. 6a, and 7a).

Due to the almost perfect coincidence of the mean values of $E_{h o m}(\theta)$ and $v_{\text {hom }}(\theta)$ with $E_{0}(\theta)$ 
and $v_{0}(\theta)$, as already discussed, Fig. 4a, 5a, 6a, 7a also represent for each angle $\theta$ the ratios between the standard deviations of $\operatorname{Ehom}_{\mathrm{h}}(\theta)$ and $v_{\text {hom }}(\theta)$ and their mean values $\bar{E}_{\text {hom }}(\theta)$ and $\bar{v}_{\text {hom }}(\theta)$, respectively.

The same variables $E_{\text {hom }}(\theta)$ and $v_{\text {hom }}(\theta)$ are also shown in Fig. $4 b, 5 b, 6 b, 7 b$ without normalization, with the homogenized elastic modulus $\mathrm{E}_{\text {hom }}(\theta)$ shown in the non-dimensional form $\sigma_{\mathrm{Ehom}}(\theta) / \mu_{\mathrm{E}}$, with $\mu_{\mathrm{E}}=500 \mathrm{MPa}$ denoting the mean value of $\mathrm{E}(\mathrm{x})$.

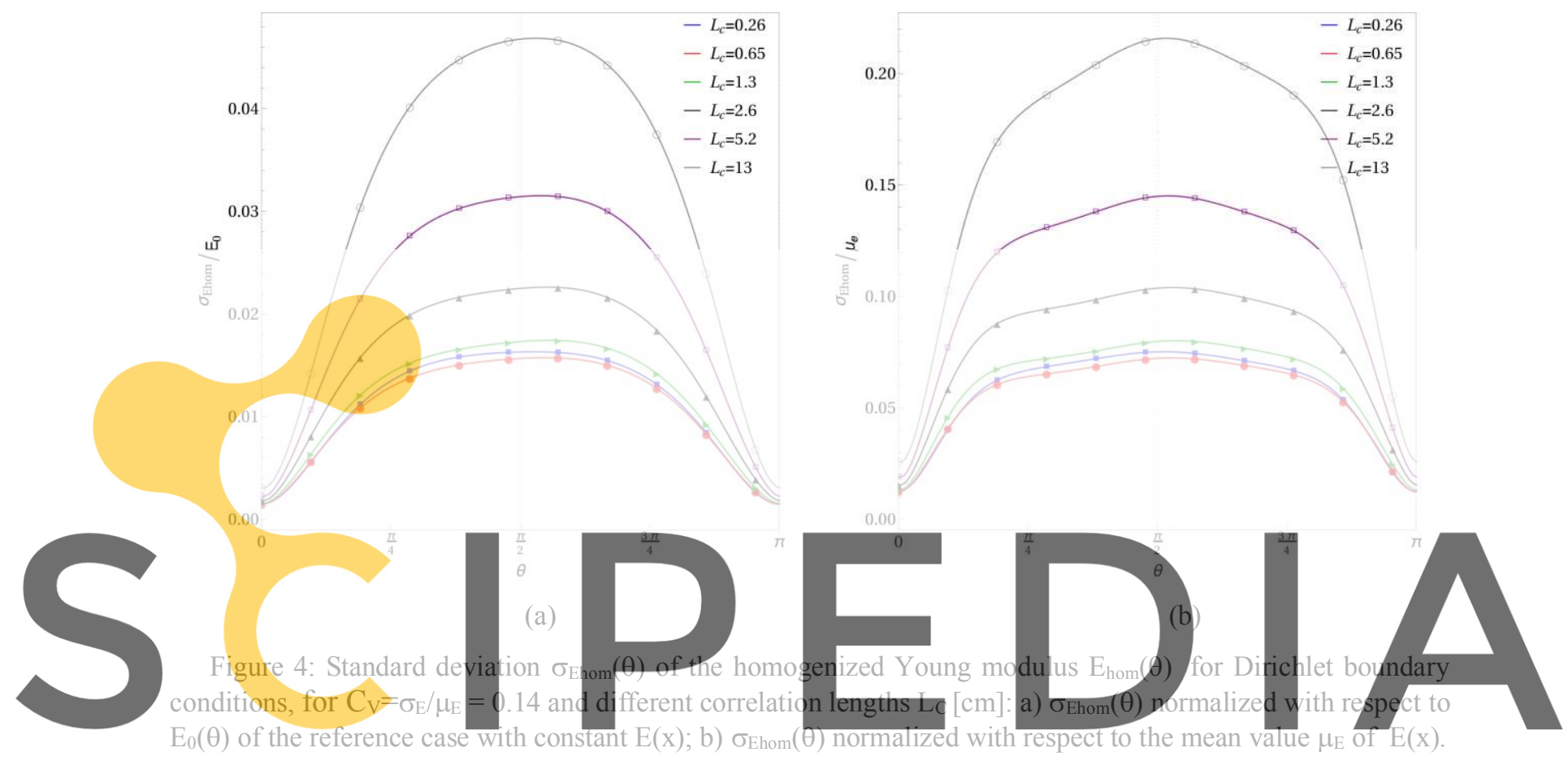

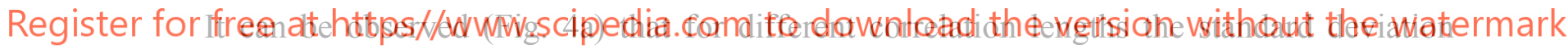

$\sigma_{\text {Ehom }}(\theta)$ of the homogenized Young modulus $\mathrm{E}_{\text {hom }}(\theta)$ is not negligible with respect to its mean value $\bar{E}_{\text {hom }}(\theta)$, with a maximum of about $5 \%$ for $L_{c}=13$ and $\theta$ around $\pi / 2$. A similar behavior is observed (Fig. 5a) for the standard deviation $\sigma_{v \text { hom }}(\theta)$ of the homogenized Poisson coefficient $v_{\text {hom }}(\theta)$.

Figures $6 \mathrm{a}-7 \mathrm{a}$ describe the dependence of the homogenized Young modulus and Poisson coefficient on $\mathrm{C}_{\mathrm{V}}=\sigma_{\mathrm{E}} / \mu_{\mathrm{E}}$, i.e. the variation coefficient of the random elastic modulus $\mathrm{E}(\mathrm{x})$ of the bed joints of the volume element in Figure 1.

As expected, the ratios between the standard deviations $\sigma_{\text {Ehom }}(\theta), \sigma_{\text {vhom }}(\theta)$ of $E_{\text {hom }}(\theta)$ and $v_{\text {hom }}(\theta)$ and their mean values $\bar{E}_{\text {hom }}(\theta)$ and $\bar{v}_{\text {hom }}(\theta)$ increase for an increasing $\mathrm{C}_{\mathrm{V}}$. Maximum values around $5 \%$ have been found for $C_{V}=\sigma_{E} / \mu_{E}=0.35$, corresponding to the limit case of a random variable $\mathrm{E}(\mathrm{x})$ oscillating between values slightly larger than zero and $2 \mu_{\mathrm{E}}$. 


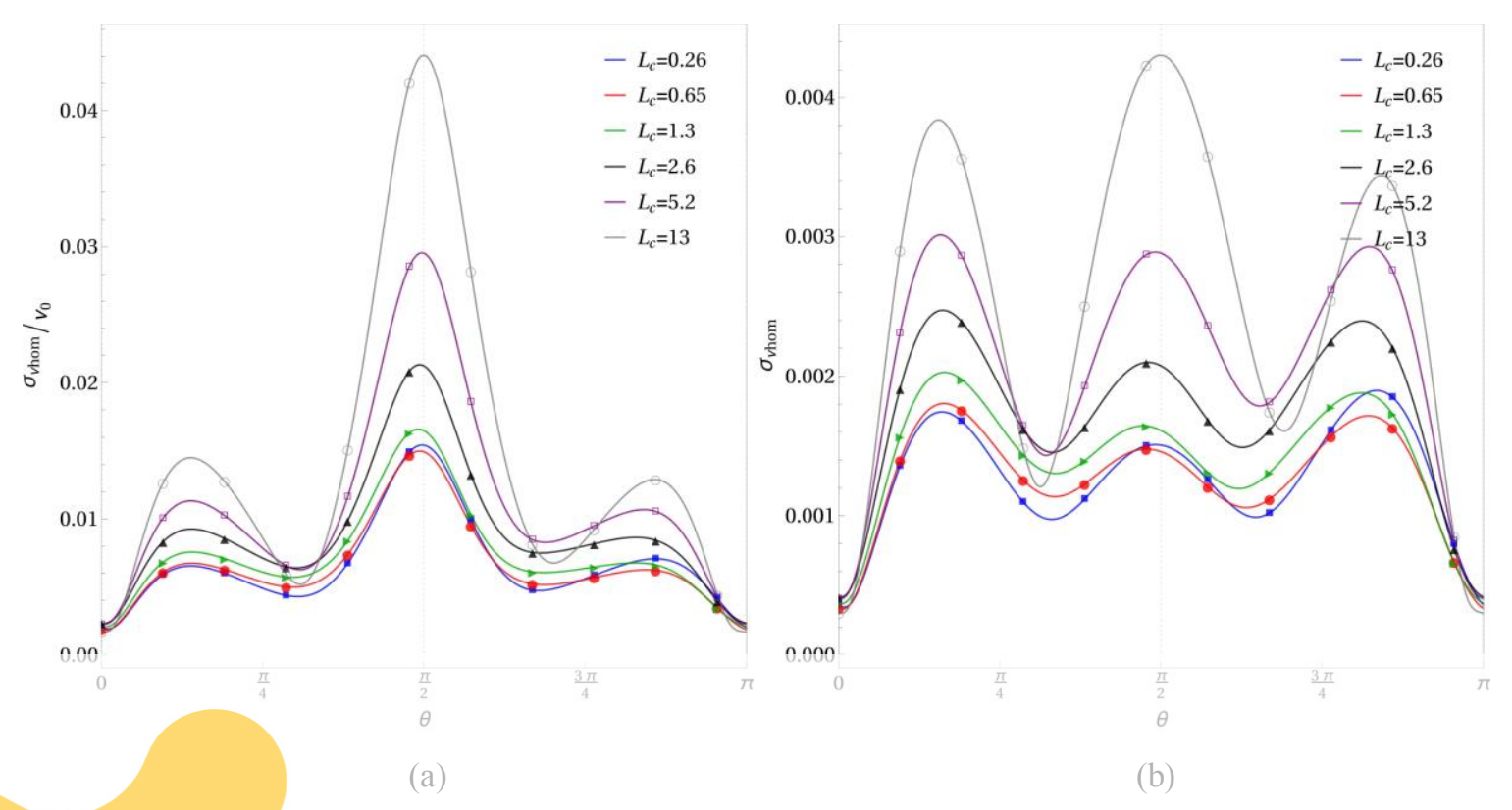

Figure 5: Standard deviation $\sigma_{\text {vhom }}(\theta)$ of the homogenized Poisson coefficient $v_{\text {hom }}(\theta)$ for Dirichlet boundary conditions, for $\sigma_{\mathrm{E}} / \mu_{\mathrm{E}}=0.14$ and different correlation lengths $\mathrm{L}_{\mathrm{C}}[\mathrm{cm}]$ : a) $\sigma_{\text {vhom }}(\theta)$ normalized with respect to $v_{0}(\theta)$ of the reference case with constant $\mathrm{E}(\mathrm{x})$; b) $\sigma_{\text {vhom }}(\theta)$.
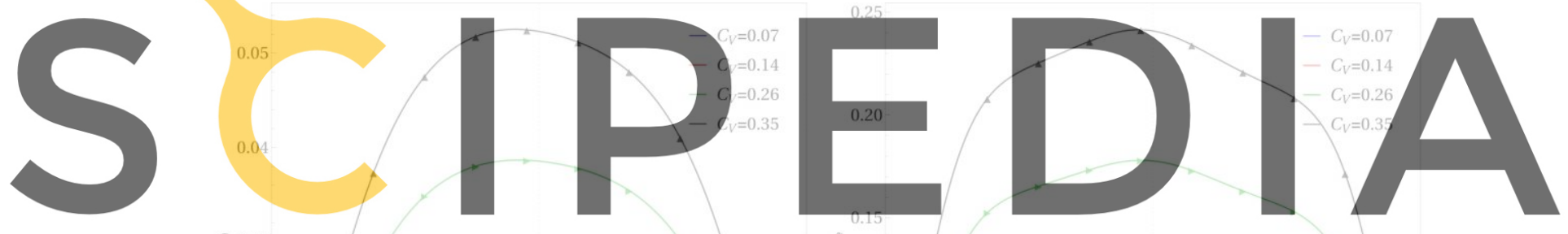

யึ 0.03

Register for free at https//www.scipedia.com to download the version without the watermark

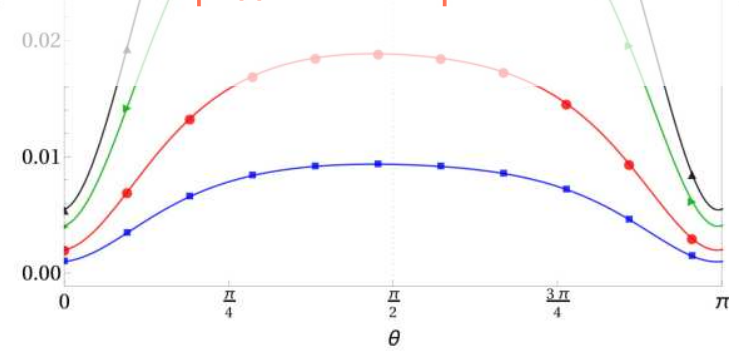

(a)

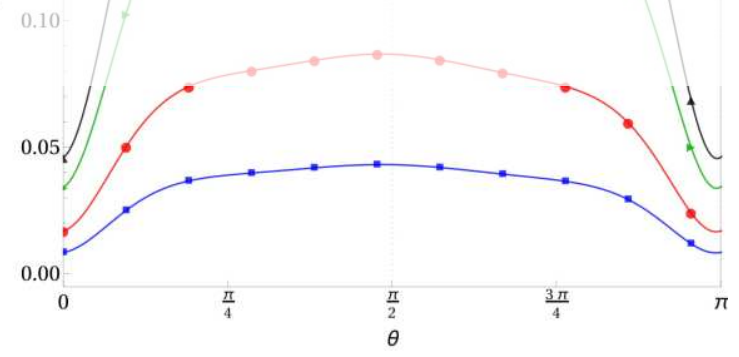

(b)

Figure 6: Standard deviation $\sigma_{\text {Ehom }}(\theta)$ of the homogenized Young modulus $E_{\text {hom }}(\theta)$ for Dirichlet boundary conditions for $L_{c}=0.26 \mathrm{~cm}$ and different ratios $C_{V}=\sigma_{E} / \mu_{E}$ : a) $\sigma_{\text {Ehom }}(\theta)$ normalized with respect to $E_{0}(\theta)$ of the reference case with constant $\mathrm{E}(\mathrm{x})$; b) $\sigma_{\mathrm{Ehom}}(\theta)$ normalized with respect to the mean value $\mu_{\mathrm{E}}$ of $\mathrm{E}(\mathrm{x})$. 


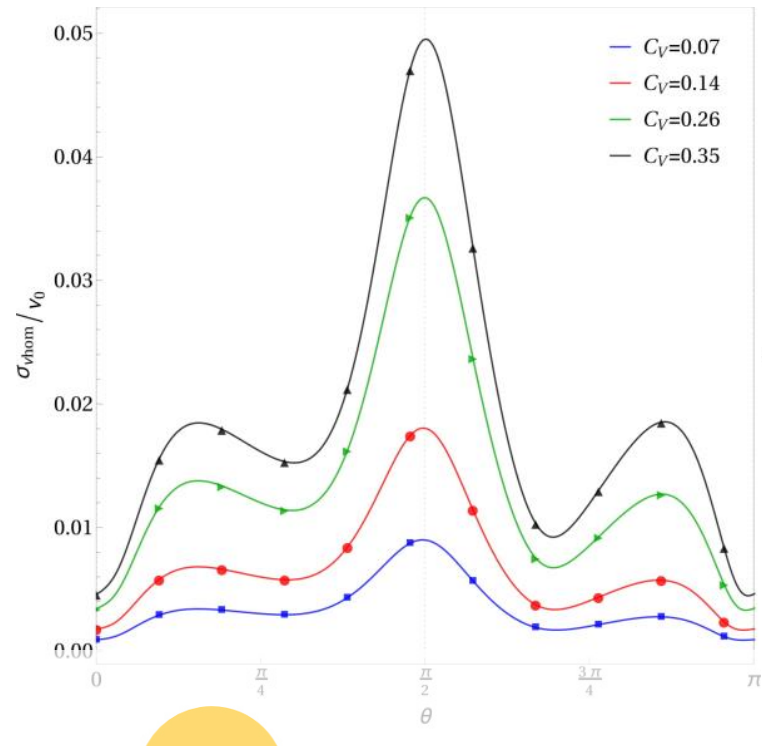

(a)

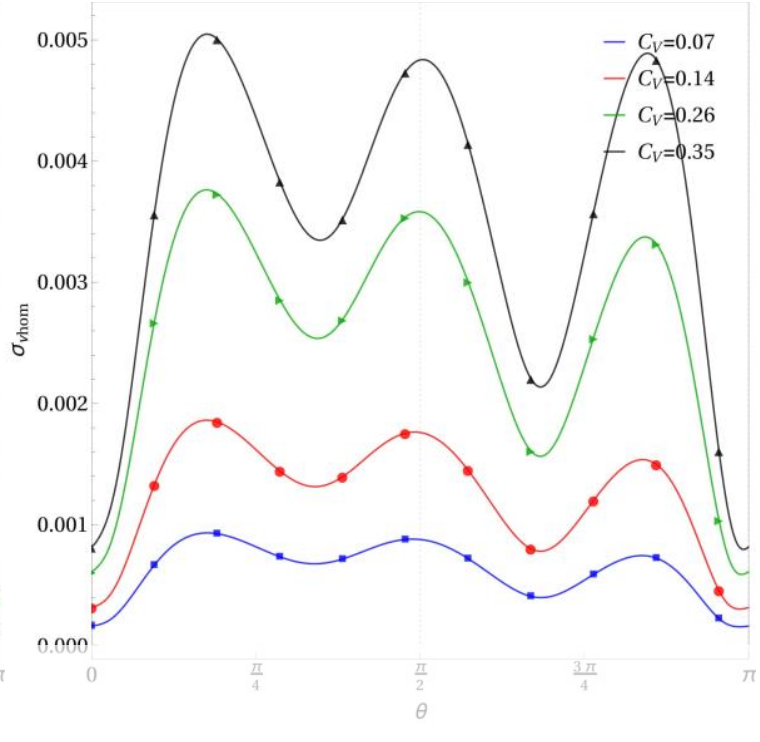

(b)

Figure 7: Standard deviation $\sigma_{\text {vhom }}(\theta)$ of the homogenized Poisson coefficient $v_{\text {hom }}(\theta)$ for Dirichlet boundary conditions for $L_{c}=0.26 \mathrm{~cm}$ and different ratios $C_{V}=\sigma_{E} / \mu_{\mathrm{E}}$ : a) $\sigma_{\text {vhom }}(\theta)$ normalized with respect to $v_{0}(\theta)$ of the reference case with constant $\mathrm{E}(\mathrm{x})$; b) $\sigma_{\text {vhom }}(\theta)$.
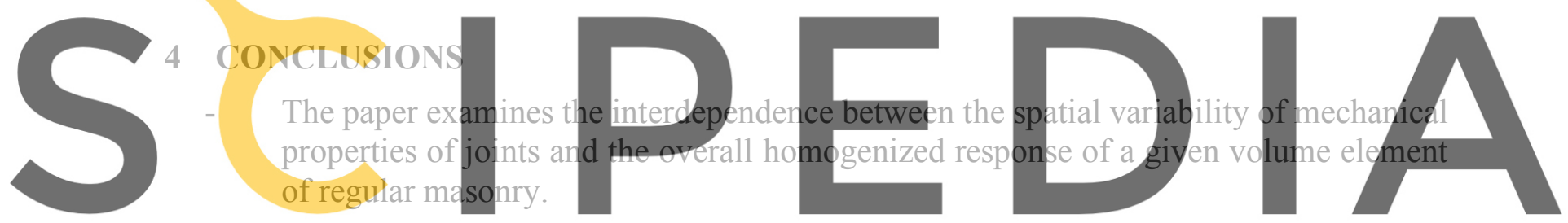

Mechanical properties of bed joints is assumed to vary according to a fixed probability

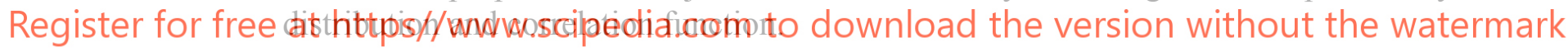

A well established computational homogenization technique is applied.

- The mechanical response, in terms of overall elastic stiffness of the selected volume element, is investigated as a function of mean, variance and correlation length of the elastic properties of the joints.

- $\quad$ As confirmed by preliminary results, the spatial variability of the mechanical properties may affect the overall response of a masonry wall.

- Such preliminary results encourage further research on historical masonry with larger spatial variability of the elastic properties.

Acknowledgements. The financial support of University "G. D'Annunzio" of Chieti-Pescara is acknowledged by the authors. 


\section{REFERENCES}

[1] L. Schueremans, D. Van Gemert, Predicting masonry properties from component properties using probabilistic techniques, Proceedings of the STRUMAS V, The Fifth International Symposium on Computer Methods in Structural Masonry, 2001.

[2] R. Zavalis, B. Jonaitis, Lourenco P.B., Analysis of bed joint influence on masonry modulus of elasticity, Proceedings of the 9 th International Masonry Conference in Guimarães, 2014.

[3] J. Li, M. J. Masia, M.S. Stewart, Stochastic spatial modelling of material properties and structural strength of unreinforced masonry in two-way bending, Structure and Infrastructure Engineering, 1-13, 2016.

[4] V. Gusella, F. Cluni, Random field and homogenization for masonry with nonperiodic microstructure, Journal of Mechanics of Materials and Structures, 1 (2): 357-386, 2006

[5] S. Ghosh, K., S. Moorthy, Two scale analysis of heterogeneous elastic plastic materials with asymptotic homogenisation and Voronoi cell finite element model. Comput Methods Appl Mech Eng 132:63-116, 1996.

[6] C. Miehe, Strain-driven homogenization of inelastic microstructures and composites based on an incremental variational formulation. Int J Numer Methods Eng 55:1285-322, 2002 .

[7] Z. Khisaeva, M. Ostoja-Starzewski, On the size of RVE in finite elasticity of random composites. J Elast 85:153-173, 2006

[8] P. Trovalusci, M.L. De Bellis, M. Ostoja-Starzewski, A. Murrali, Particulate random composites homogenized as micropolar materials. Meccanica 49, 2719-2727, 2014.

[9] D. Tromans, Elastic anisotropy of hcp metal crystals and polycrystals, International Journal of Research and Reviews in Applied Sciences, 6.4, pp.462-463, 2011.

[10] A. Bacigalupo, M.L. De Bellis, Auxetic anti-tetrachiral materials: Equivalent elastic properties and frequency band-gaps, Composite Structures, 131: 530-544, 2015.

[11] C.W. Gardiner, Handbook of Stochastic Methods (2nd ed.), Springer-Verlag, p. 106, ISBN 978-0-387-15607-1, 1985 